\title{
Adsorption of Phenol on Gold As Studied by Capacitance and Reflectance Measurements
}

\author{
R. O. Lezna, N. R. de Tacconi, S. A. Centeno, and A. J. Arvia* \\ Instituto de Investigaciones Fisicoquimicas Teóricas y Aplicadas (INIFTA), Facultad de \\ Ciencias Exactas, Universidad Nacional de La Plata, Sucursal 4, Casilla de Correo 16, \\ 1900 La Plata, Argentina
}

Received May 3, 1990. In Final Form: September 27, 1990

\begin{abstract}
Adsorption of phenol on a polycrystalline gold electrode has been studied by capacitance and modulated reflectance measurements. Two main processes were detected. First, an adsorption-desorption peak was observed at negative charge on the electrode which was related to a flat configuration of the molecule bonded to the surface through its $\pi$ system. In this case the interactions between adsorbed phenol and the electrode are not strong enough to modify the intrinsic optical properties of the metal. A second capacitive peak, detected over a region of positive charge on the metal, was found to show hysteresis and, besides, it distorts the electroreflectance effect of gold to such an extent that separation of the optical effects of charge and coverage was not possible. This behavior, typical of the formation of strong covalent bonds, was associated with the reorientation of the molecule from flat to vertically attached to the metal by means of its oxygen atom.
\end{abstract}

\section{Introduction}

Fundamental work on the adsorption of organic substances on solid metal electrodes is of interest in relation to the changes it produces at the electrochemical interface and the influence of adsorbate coverage, orientation, and properties of the surface bonding on the mechanism of electrochemical reactions. ${ }^{1}$

In contrast to adsorption studies on $\mathrm{Hg}$ electrodes, the number of contributions on the adsorption of organic molecules on solid electrodes is rather small, although it has been increasing relatively rapidly in the last decade. This is due, at least in part, to the new perspectives offered by in situ spectroscopic methods and surface preparation techniques which have become useful and reliable to complement the characterization of the electrode/solution interface. . $^{-4}$

Capacitance and in situ optical information about the adsorption of phenol on gold electrodes seems to be scarce despite the fact that the properties of the adsorption of phenol are of interest for its elimination in wastewater treatments. 5

This paper deals with the study of the adsorption of phenol on polycrystalline gold by differential capacity and in situ visible reflectance spectroscopy.

Capacitance curves were obtained with a view to determining the surface coverage by adsorbates, the potential ranges associated with the various stages of the adsorption process, the adsorption energy, and the likely orientation of the adsorbed phenol molecules.

Optical measurements, in the form of differential curves in the visible region, proved to be useful to describe the different degree of interaction between the gold and the phenol molecule in the two adsorption stages observed, as

(1) Hubbard, A. T.; Stickney, J. L.; Soriaga, M. P.; Chia, V. K. F. Rosasco, S. D.; Schardt, B. C.; Solomun, T.; Song, D.; White, J. H.; Wieckowski, A. J. Electroanal. Chem. Interfacial Electrochem. 1984, 168, 43

(2) Kolb, D. M. In Trends in Interfacial Electrochemistry; Silva, A F., Ed.; Reidel: Dordrecht, 1986.

(3) Bewick, A.; Pons, S. In Advances in Infrared and Raman Spectroscopy; Clark, R. J. H., Hester, R. E., Eds.; Wiley-Heyden: Chichester, 1985; Vol. 12, p 1.

(4) Sakamaki, K.; Matsunaga, S.; Itoh, K.; Fujishima, A.; Gohshi, Y. Surf. Sci. 1989, 219, L531.

(5) Sharifian, H.; Kirk, D. W. J. Electrochem. Soc. 1986, 133, 921. shown by the change in the electroreflectance spectrum of gold as a function of coverage by phenol. Processes arising from strongly adsorbed species were particularly better resolved by optical measurements than by capacitance curves.

\section{Experimental Section}

Optical and electrochemical experiments were carried out at room temperature by using Kel-F encased Au (99.99\%) disks of $7 \mathrm{~mm}$ diameter. Prior to use the electrode was mechanically polished with alumina of different grades, down to $0.05 \mu \mathrm{m}$, and cleaned in an ultrasonic bath. A $0.1 \mathrm{M} \mathrm{NaF}$ (Merck pa) solution was employed as supporting electrolyte unless otherwise stated. Solutions for adsorption measurements were prepared with triple distilled Milli-Q water that had been previously deoxygenated with purified nitrogen. The concentration of phenol (Merck pa) ranged from $2 \times 10^{-5}$ to $5 \times 10^{-3} \mathrm{M}$. Higher bulk phenol concentrations were not employed as they shifted the electrodesorption process into the HER potential region. All potentials were measured and are quoted against a MSE.

Capacitance/potential $(C-E)$ measurements were carried out at $11 \mathrm{~Hz}$, by using a two-phase lock-in amplifier.

Potential modulated (differential) reflectance spectra were measured, in situ, either by scanning the wavelength at constant bias potential or by slowly sweeping the potential at a given wavelength. The experimental setup has been described elsewhere. ${ }^{6}$ Cyclic voltammetry was carried out with a conventional setup.

\section{Results and Discussion}

Electrochemical Measurements. Cyclic voltammograms were run as the first measurements of the system in order to check on cleanliness of both the electrode surface and solution and to ascertain the size of the potential window free from (pseudo)faradaic reactions. Figure 1 displays voltammograms for the polycrystalline gold in the absence (a) and in the presence (b) of phenol with different upper potential limits so as to include (or exclude) the formation of gold oxide. Voltammogram a, corresponding to the supporting electrolyte, was found to show a reproducible profile after a few cycles. Its shape

(6) Lezna, R. O.; de Tacconi, N. R.; Arvia, A. J. J. Electroanal. Chem. Interfacial Electrochem. 1988, 255, 251. 


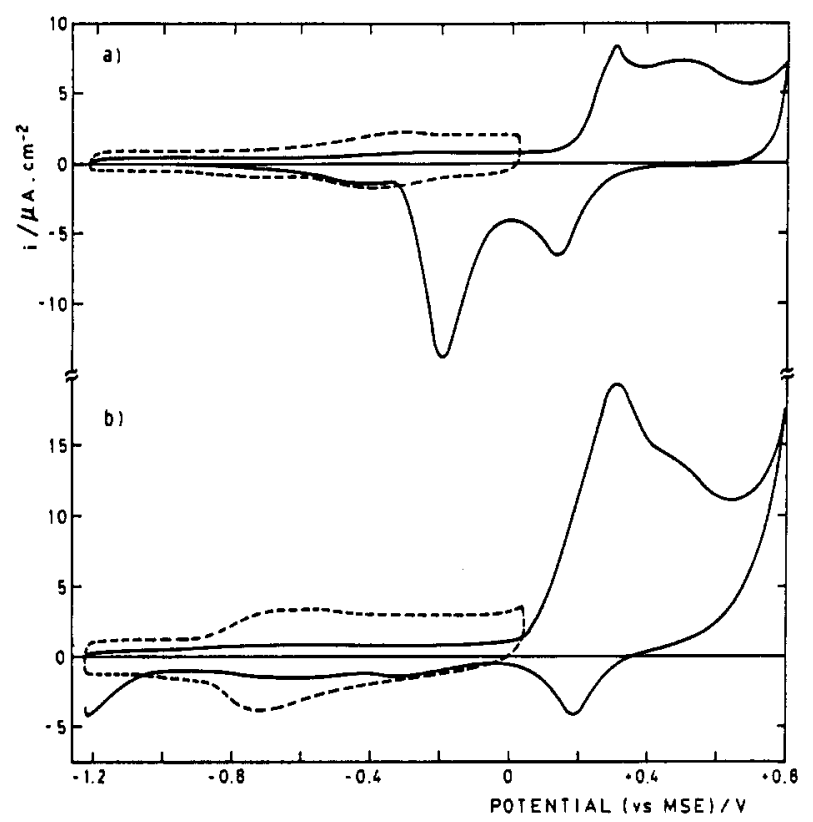

Figure 1. Voltammograms for polycrystalline gold in $0.1 \mathrm{M} \mathrm{NaF}$ at $50 \mathrm{mV} / \mathrm{s}$ in the supporting electrolyte (a) and in the presence of $1 \times 10^{-3} \mathrm{M}$ phenol (b): initial potential, $-1.2 \mathrm{~V}$; upper limits, +0.8 and $+0.050 \mathrm{~V}$. Current density scales correspond to solid lines. Broken line curves have been enlarged a factor of 2.5 in part $a$ and a factor of 4 in part $b$.

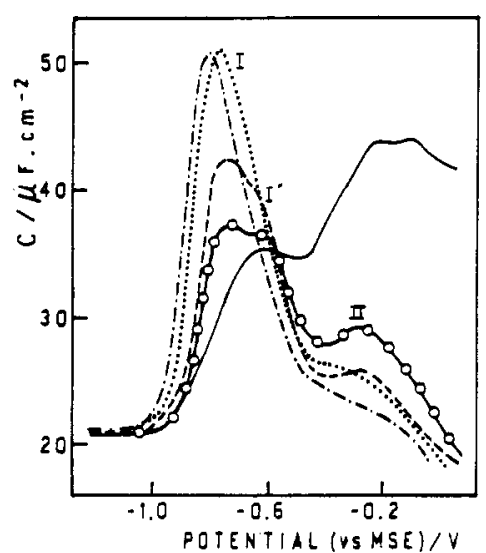

Figure 2. Differential capacitance for gold in $0.1 \mathrm{M} \mathrm{NaF}(\longrightarrow)$ and in the presence of the following phenol concentrations: $(-0-)$ $5.0 \times 10^{-5} \mathrm{M},(--) 1.5 \times 10^{-4} \mathrm{M},(\cdots) 1.0 \times 10^{-3} \mathrm{M},(-.-) 3.0$ $\times 10^{-3} \mathrm{M}$; sweep rate, $2.3 \mathrm{mV} / \mathrm{s} ;$ modulation frequency $f=11 \mathrm{~Hz}$; amplitude, $2.8 \mathrm{mV}$ (rms).

coincides with data previously reported. ${ }^{7}$ In the presence of phenol, curve $b$, a double layer region between -1.2 and $0.050 \mathrm{~V}$ exhibits the adsorption/desorption peaks characteristic of phenol electroadsorption. When the upper limit was made more positive, up to $0.8 \mathrm{~V}$, the onset of phenol oxidation was observed at ca. $+0.050 \mathrm{~V}$, that is outside the working region which was limited on the positive potential side to $0 \mathrm{~V}$. At the other end, $-1.2 \mathrm{~V}$, no faradaic reduction could be detected either in the presence of phenol or in the supporting electrolyte, provided the positive reversal potential was kept at 0.050 $\mathrm{V}$.

Capacity curves for phenol concentrations ranging from $5 \times 10^{-5}$ to $3 \times 10^{-3} \mathrm{M}$, Figure 2, show two main peaks, I and II, and a shoulder, $\mathrm{I}^{\prime}$, on the positive side of peak I. The capacity curve in $0.1 \mathrm{M} \mathrm{NaF}$ exhibits a diffuse layer minimum at $-0.48 \mathrm{~V}$. This value corresponds to the

(7) Nguyen Van Huong, C.; Hinnen, C.; Dalbera, J. P.; Parsons, R. J. Electroanal. Chem. Interfacial Electrochem. 1981, 125, 177.

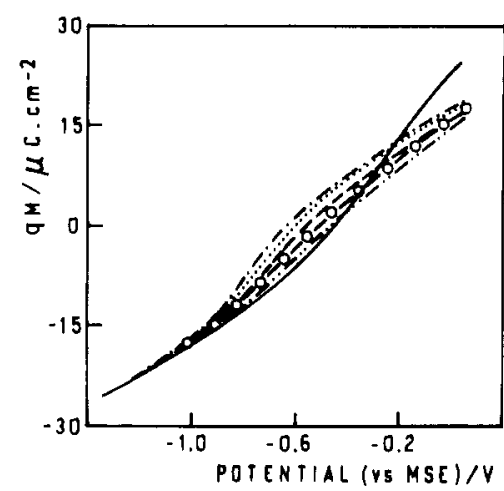

Figure 3. Charge density vs potential curves for $0.1 \mathrm{M} \mathrm{NaF}$ $(-)$ and for phenol concentrations as follows: $(-\cdots-) 2.0 \times$ $10^{-5} \mathrm{M} ;(-0-) 5.0 \times 10^{-5} \mathrm{M} ;(--) 1.5 \times 10^{-4} \mathrm{M} ;(.) .1.0 \times 10^{-3}$ $\mathrm{M} ;(-\cdot) \mathbf{3 . 0} \times 10^{-3} \mathrm{M}$.

potential of zero charge (pzc) and coincides with reported data. ${ }^{8}$ Capacity curves for every concentration of phenol merge with that of the base electrolyte at potentials more negative than $-1.2 \mathrm{~V}$, indicating that no adsorption of phenol is taking place in this region.

The adsorption-desorption peak I shows a capacitive behavior of the type found in the adsorption of neutral organic molecules in that its position is shifted to negative potentials and the peak height increases as the phenol concentration in solution is increased.

The process giving rise to shoulder $\mathrm{I}^{\prime}$ becomes better defined at low phenol concentration $\left(10^{-6} \mathrm{M}\right)$ and its position is shifted negatively in a magnitude similar to that displayed by peak I with increasing phenol concentration. However the increase in the contribution of shoulder $I^{\prime}$ with phenol concentration is less than the corresponding change of peak $I$. As a result shoulder $I^{\prime}$ becomes masked by peak I for phenol concentrations greater than $1 \times 10^{-3} \mathrm{M}$. Shoulder I' might reflect the nonhomogeneous character of the polycrystalline gold surface even though the molecule is not sufficiently small to probe properly the atomic features of the surface.

Peak II, which is better defined at low concentrations, is broader and smaller than peak I. Its contribution decreases with bulk phenol concentration.

Charge densities, $q_{\mathrm{M}}$, were obtained through integration of the $C-E$ curves and are displayed in Figure 3 as a function of potential, the initial charge value at $-1.2 \mathrm{~V}$ being that of the supporting electrolyte taking into account that all of the curves coincide at this potential. The pzc was found to shift to negative potentials with increasing concentrations of phenol on the surface. This behavior has also been reported for pyridine on gold $^{9}$ and other aromatic compounds on $\mathrm{Hg}^{10}$ The magnitude of the effective dipole moment of the adsorbed phenol molecule can be estimated from the shift of the pzc from zero coverage to maximum coverage, which is given by the equation

$$
\delta E_{\mathrm{pzc}}=4 \pi \Gamma_{\max }\left(\mu^{\mathrm{phe}}-n \mu^{\mathrm{w}}\right) / \epsilon
$$

where $\mu^{\text {phe }}$ and $\mu^{\mathrm{w}}$ are the components of the dipole moments, normal to the surface, of phenol and water molecules, respectively, $\epsilon$ is the dielectric constant of the inner part of the double layer, and $n$ is the number of water molecules displaced by one adsorbed phenol mol-

(8) Hinnen, C.; Nguyen Van Huong, C.; Rousseau, A.; Dalbera, J. P. J. Electroanal. Chem. Interfacial Electrochem. 1979, 95, 131.

(9) Stolberg, L.; Ritcher, J.; Lipkowski, J.; Irish, D. E. J. Electroanal. Chem. Interfacial Electrochem. 1986, 207, 213.

(10) Damaskin, B. B.; Petrii, O. A.; Batrakov, B. B. Adsorption of Organic Compounds at Electrodes; Plenum Press: New York, 1971. 


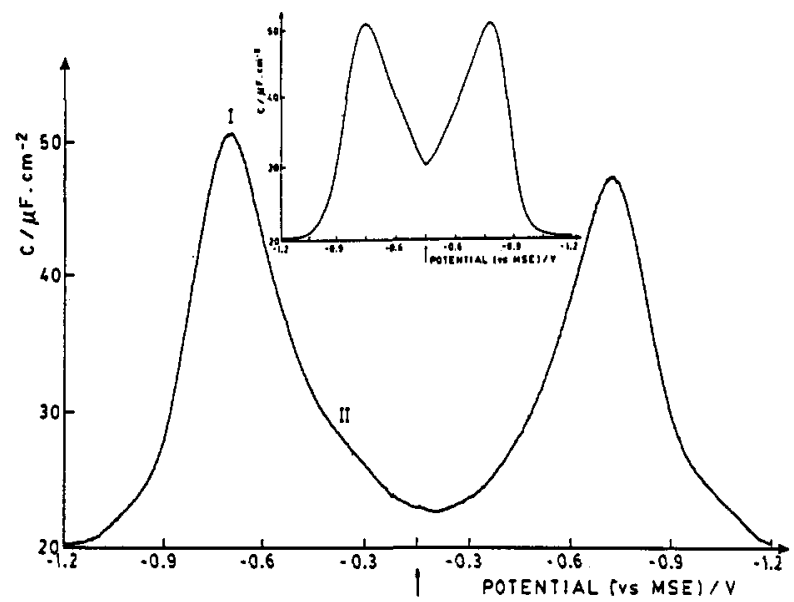

Figure 4. Differential capacitance curves for gold in $0.1 \mathrm{M} \mathrm{NaF}$ $+1 \times 10^{-3} \mathrm{M}$ phenol: $f=11 \mathrm{~Hz}$; amplitude $=2.4 \mathrm{mV}(\mathrm{rms})$; sweep rate $=2.2 \mathrm{mV} / \mathrm{s}$; initial potential, $-1.2 \mathrm{~V}$; reversing potential, $-0.15 \mathrm{~V}$ and $-0.45 \mathrm{~V}$ (inset). The reversing potential is indicated by the arrow on the potential axis.

ecule. ${ }^{11}$ The negative shift of pzc suggests that $\left|n \mu^{\mathrm{w}}\right|<$ $\left|\mu^{\text {phe }}\right|$ with $\mu^{\text {phe }}<0$. The dipole moment of the isolated phenol molecule is in the plane of the aromatic ring. However the adsorbed molecule may develop asymmetric charge distributions as a result of interactions with both the metal and solvent molecules on the solution side. Thus, a flat orientation may give rise to asymmetric interactions between the $\pi$ electrons and the metal, with transfer of electrons from the molecule to the metal, leading to an induced dipole moment with a direction consistent with that required by the pzc shift. The same dipole moment direction results for a vertical configuration of the adsorbed molecule with the lone pair of the oxygen overlapping empty states on the metal. The most likely orientation will be proposed later, on the basis of the value found for $\Gamma_{\max }$.

Charge values calculated for potentials more positive than ca. $-0.4 \mathrm{~V}$, region spanned by peak II, should be taken with caution as the capacity curve displays some hysteresis on reversing the potential sweep (scan rate $2.2 \mathrm{mV} / \mathrm{s}$ ), Figure 4. This behavior, not observed in other regions, may be indicating that equilibrium has not been reached in this zone under the experimental conditions employed. Therefore data analysis was carried out only up to -0.45 $\mathrm{V}$.

Plots of surface pressure as a function of potential, Figure 5 , were obtained by subtracting the curve corresponding to the relative interface tension of a phenol-containing solution from that of the phenol-free supporting electrolyte. The bell shape is characteristic of the adsorption of neutral organic molecules, the relatively high values of $\pi$ being similar to those reported for aniline on gold.12 Surface pressure values were plotted as a function of $\ln$ $c$ at constant potentials, up to $-0.45 \mathrm{~V}$, that is, excluding the region of process II. The curves were then shifted along the $\ln c$ axis in order to obtain a composite curve, Figure 6, according to the method suggested by Parsons. ${ }^{13}$ Therefore the curves $\pi$ vs $\log c$ are congruent with respect to the electrode potential: The maximum surface excess determined through differentiation of this curve, for the highest phenol concentration employed, led to a value for $\Gamma_{\max }$ of ca. $3.0 \times 10^{-10} \mathrm{~mol} / \mathrm{cm}^{2}$. This value is close to that

(11) Parsons, R. In Modern Aspects of Electrochemistry; Bockris, J. O'M., Ed.; Butterworths: London, 1954; Vol. 1, p 103.

(12) Nguyen Van Houng, C. J. Electroanal. Chem. Interfacial Electrochem. 1989, 264, 247.

(13) Parsons, R. Proc. R. Soc. London, Ser. A 1961, 261, 79.

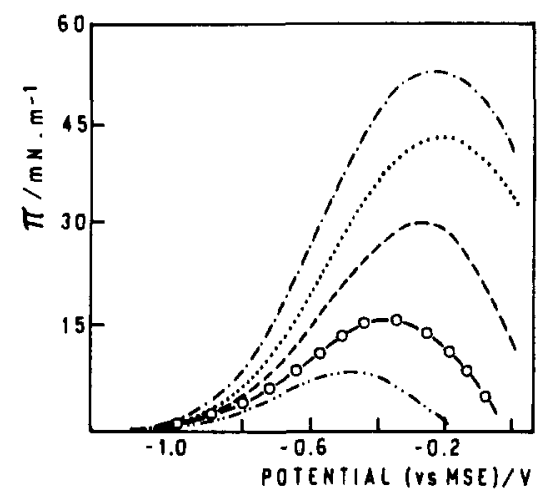

Figure 5. Surface pressure vs potential curves in $0.1 \mathrm{M} \mathrm{NaF}$ containing different amounts of phenol as follows: $(-\cdots-) 2.0 \times$ $10^{-5} \mathrm{M} ;(-0-) 5.0 \times 10^{-6} \mathrm{M} ;(---) 1.5 \times 10^{-4} \mathrm{M} ;(\cdots) 1.0 \times 10^{-3}$ $\mathrm{M} ;(-\bullet-) 3.0 \times 10^{-3} \mathrm{M}$.

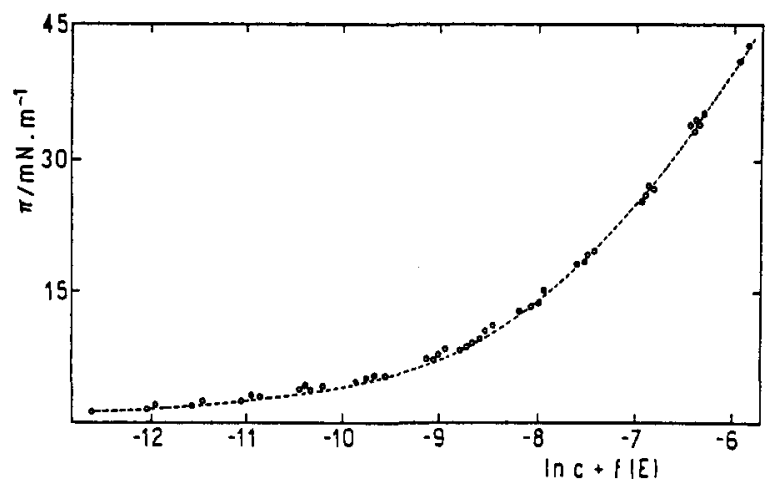

Figure 6. Composite surface pressure calculated at constant potentials.

expected for a flat orientation of the phenol molecule on the surface, $3.8 \times 10^{-10} \mathrm{~mol} / \mathrm{cm}^{2}$, taking the projected area of the molecule to be $43 \AA^{214}$ in that position. On the contrary a vertical orientation, with projected area equal to $21 \AA^{2}$, would lead to a $\Gamma_{\max }$ of $7.9 \times 10^{-10} \mathrm{~mol} / \mathrm{cm}^{2}{ }^{15} \mathrm{It}$ should be noticed that flat orientation was also found for phenol on $\mathrm{Pt},{ }^{16}$ aniline on gold, ${ }^{12}$ pyridine on $\mathrm{Ag}, \mathrm{Cu}$, and $\mathrm{Ni}, 17,18$ and pyridine on $\mathrm{Au}^{9}$ even at rather negative potentials.

The plot of $\ln \{c(1-\theta) / \theta\}$ vs $\theta$ (Frumkin isotherm) $)^{10}$ in the potential region of peak I led to values for the interaction constant $a$ of -1.4 (repulsive interactions) and for adsorption energy, $\delta G$, of $-29 \mathrm{~kJ} / \mathrm{mol}$. The free-energy of adsorption was calculated from the adsorption coefficient through the equation $\delta G=-R T \ln (55.5 \beta)$. This value was subsequently corrected for particle-particle interactions by taking $\theta=0$ as the reference state for the adsorbate and unit mole fraction for the solute in solution. ${ }^{19}$ The concentration of phenol in solution necessary to obtain a given coverage on gold is less than that required for the adsorption of phenol on $\mathrm{Hg} .{ }^{16}$ This is consistent with the adsorption energy for phenol on Au being higher than the corresponding value for phenol on $\mathrm{Hg}$. Similar findings were reported for the adsorption of pyridine and aniline on $\mathrm{Au} .{ }^{9,12}$

(14) Blomgren, E.; Bockris, J. O'M.; Jesch, C. J. Phys. Chem. 1961, 65, 2000.

(15) Hansen, R. S.; Kelsh, D. J.; Grantham, D. H. J. Phys. Chem. 1963, $67,2316$.

(16) Lu, F.; Salaita, G. N.; Laguren-Davidson, L.; Stern, D. A.; Wellner, E.; Frank, D. G.; Batina, N.; Zapien, D. C.; Walton, N.; Hubbard, A. T. Langmuir 1988, 4, 637.

(17) Conway, B. E.; Barradas, R. G.; Zawidzky, T. J. Phys. Chem. $1958,62,676$.

(18) Barradas, R. G.; Conway, B. E. J. Electroanal. Chem. 1963, 6, 314

(19) Jastrzebska, J.; Jurkiewicz-Herbich, M.; Trasatti, S. J. Electroanal. Chem. 1987, 216, 21. 


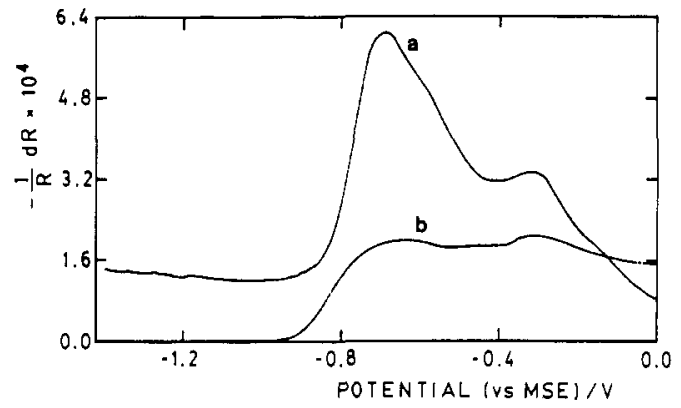

Figure 7. Differential reflectivity vs potential curves taken at $2.5 \mathrm{eV}$ (a) and $1.7 \mathrm{eV}(\mathrm{b}): \phi=59^{\circ} ;$ p-polarization; $f=11 \mathrm{~Hz}$; amplitude $=18 \mathrm{mV}$ (rms).

Phenol is likely to form hydrogen bonding in the adsorbed state with solvent molecules on the solution side leading to a highly structured configuration. This arrangement should not be significantly perturbed by the structure breaking properties of the fluoride ion as the concentration of this anion should be considerably decreased in the interface of a negatively charged electrode.

Reflectance Measurements. Differential reflectivity curves for $1 \times 10^{-4} \mathrm{M}$ phenol are presented in Figure 7 for two photon energies, namely $2.5 \mathrm{eV}$, absorption edge of gold, and $1.7 \mathrm{eV}$, where there is no contribution from interband transitions in the metal.

At $2.5 \mathrm{eV}$, the optical response is similar in shape to that of the capacity curve obtained under comparable experimental conditions, Figure 2, although in the reflectivity curve the features related to peak II are more clearly resolved. At potentials more negative than $-1.1 \mathrm{~V}$ only the electroreflectance effect of bare gold can be observed, whereas in the potential region spanning peaks I and I the phenol coverage modulation leads, in turn, to the modulation of the metal charge, and consequently to an increase in the optical response. On the other hand, at 1.7 $\mathrm{eV}$ the curve is distinctly different from that obtained at $2.5 \mathrm{eV}$. Thus peaks I and $\mathrm{I}^{\prime}$ are attenuated, whereas the intensity of peak II is comparatively much less reduced, indicating a strong interaction of the species with the substrate.

The spectral response taken at the potential of peak I is similar in shape to that of bare gold, ${ }^{20}$ although its intensity is enhanced (made more negative) by the presence of phenol on the surface. This change in intensity was found to be proportional to the total charge on the metal, Figure 8.

On the other hand in the region of process II the spectral response is clearly distorted. Changes in intensity (enhancement) and shape are observed with respect to those expected for bare gold, Figure 9 . This behavior is to be expected if a strong covalent bond is formed between the adsorbate, presumably as phenoxide ion, and the gold surface. This possibility is borne out by the fact that peak II is much better defined in alkaline solutions, Figure 10.

Maximum II, which is more easily observed in the red region of the spectrum, beyond ca. $650 \mathrm{~nm}$, is less dependent on frequency than are peaks I and shoulder $I^{\prime}$ (Figure 9). According to this behavior the response of peak II seems to arise mainly from the electroreflectance effect of gold modified by the presence of the adsorbate, whereas the optical response corresponding to both peaks I and I' stems mainly from the modulation of coverage. Further support for this assumption is provided by the fact that the process giving rise to peak II can be more easily observed, in the red region, through differential re-

(20) McIntyre, J. D. Symp. Faraday Soc. 1970, 4, 99.

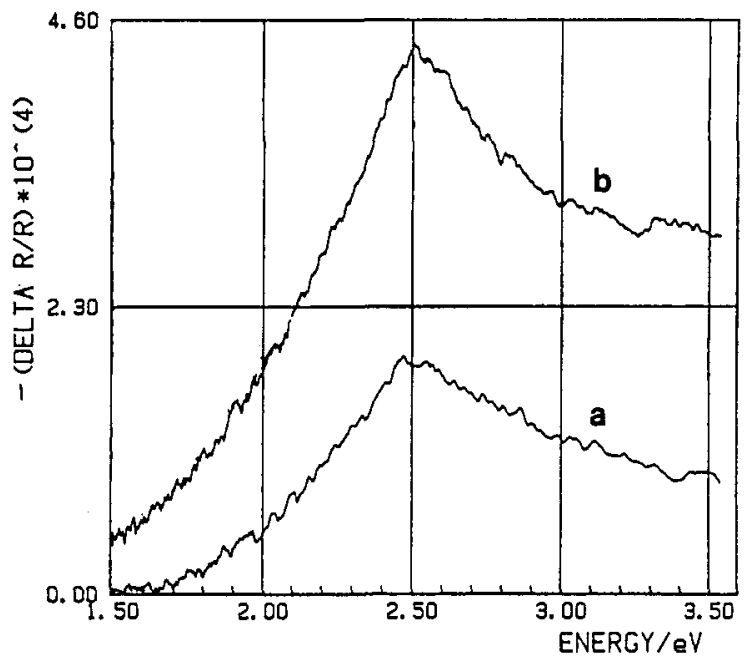

Figure 8. Differential reflectance spectra measured at $-0.72 \mathrm{~V}$ (peak I) in the base electrolyte $(a)$ and in the presence of $1 \times 10^{-4}$ $\mathrm{M}$ phenol (b): $\phi=59^{\circ} ; \mathrm{p}$-polarization; $f=11 \mathrm{~Hz}$; amplitude $=$ $18 \mathrm{mV}$ (rms).

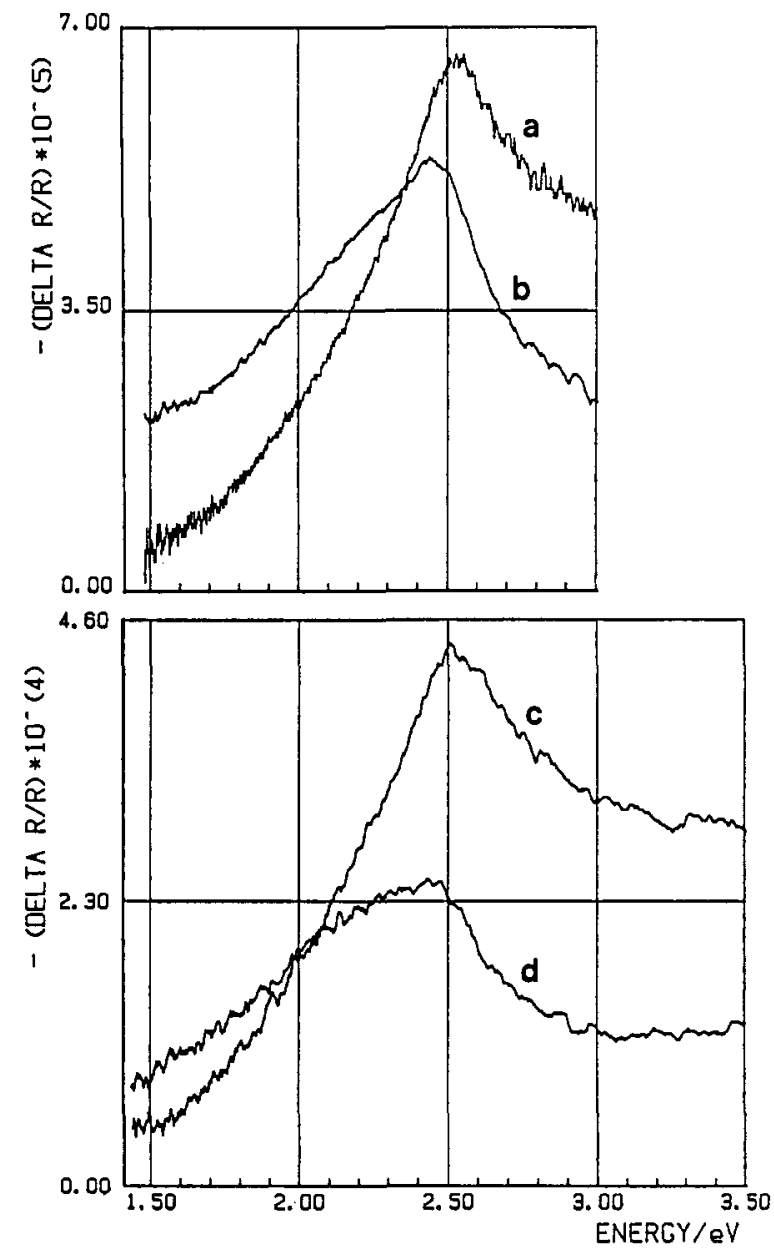

Figure 9. Frequency dependence of the differential reflectance spectra obtained at $-0.72 \mathrm{~V}$ (peak I) and $-0.32 \mathrm{~V}$ (peak II): (a) $440 \mathrm{~Hz},-0.72 \mathrm{~V}$; (b) $440 \mathrm{~Hz},-0.32 \mathrm{~V}$; (c) $11 \mathrm{~Hz},-0.72 \mathrm{~V}$; (d) 11 $\mathrm{Hz},-0.32 \mathrm{~V} ; \mathrm{p}$-polarization; $\phi=59^{\circ} ;$ amplitude $=18 \mathrm{mV}(\mathrm{rms})$.

flectivity measurements than through electrical determinations such as capacitance measurements.

Some organic molecules are known to undergo a reorientation process in the potential region corresponding to peak II. ${ }^{9}$ Therefore, in the case of phenol on gold the transformation leading to this peak can be associated with the reorientation of the molecule from flat to vertical. The transition between the two orientations is not abrupt in 


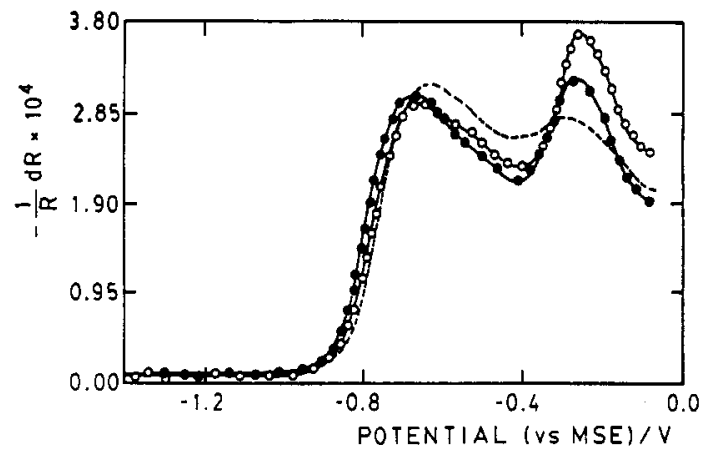

Figure 10. $\mathrm{pH}$ dependence of the reflectivity vs potential curves at $1.7 \mathrm{eV}$ for $2 \times 10^{-3} \mathrm{M}$ phenol: $(---) \mathrm{pH}=6,(-\cdot-) \mathrm{pH}=9$, $(-0-) \mathrm{pH}=11 ; \phi=59^{\circ} ; \mathrm{p}$-polarization; $f=11 \mathrm{~Hz}$; amplitude = $18 \mathrm{mV}$ (rms).

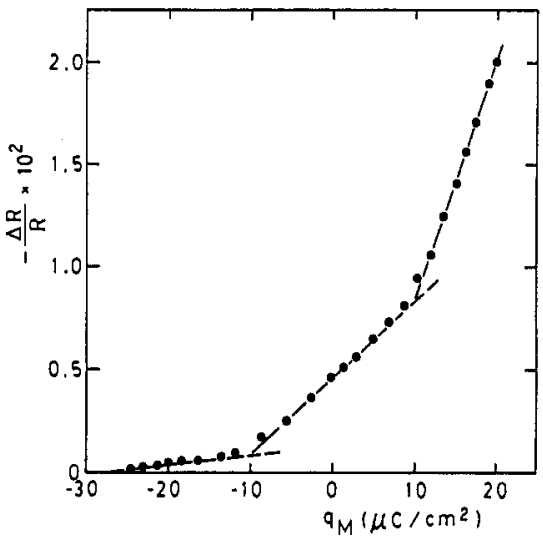

Figure 11. Plot of integral reflectance against charge densities for $1.7 \times 10^{-3} \mathrm{M}$ phenol: initial potential, $-1.3 \mathrm{~V}$. Final potentials were stepwise changed from -1.2 to $0.05 \mathrm{~V}$. Charge density for each potential was determined by integration of differential capacity.

this case, particularly at high bulk concentrations of phenol, pointing to the existence of interactions between adsorbed molecules in the two orientations. In this position the bonding of the molecule to the surface can be thought of as taking place either through the lone pair of the oxygen atom or, at higher $\mathrm{pH}$, by means of the phenoxide ion, taking into account the surface is positively charged in this potential region.

The optical response from integral reflectance measurements taken at $1.7 \mathrm{eV}$ and for $1.7 \times 10^{-3} \mathrm{M}$ phenol away from the absorption edge of gold is presented in Figure 11. The curve can be separated into three linear zones when plotted against the total charge on the metal.

In Figure 11, the first linear zone corresponding to negative charges up to $\mathrm{ca} .-10 \mu \mathrm{C} / \mathrm{cm}^{2}$ arises from the electroreflectance effect of bare gold, its contribution being small as it results mainly from the free electrons of the metal.

The second region, covering the adsorption-desorption process of phenol and made up of processes $I$ and $I^{\prime}$, displays a slope higher than that observed in the first zone and was found to be linear with both the total charge on the metal and the fractional coverage of phenol, Figure 12. This behavior is to be expected if the contribution of charge and coverage to the reflectance can be separated out. ${ }^{21}$ The relationship between $\delta R / R$ and $\theta$ was obtained by subtracting the response of the metal in the phenolfree supporting electrolyte from the curve measured with phenol in solution. The degree of surface coverage, $\theta$, was calculated in this case following the Frumkin model in

(21) Bewick, A.; Robinson, J. Surf. Sci. 1976, 55, 349.

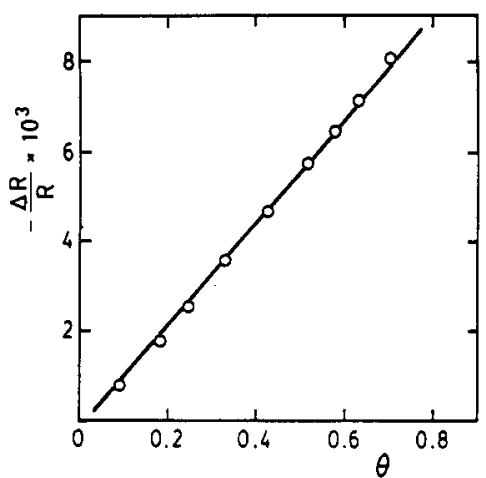

Figure 12. $\delta R / R$ vs $\theta$ plots in the potential region of peak $I$ for $1.7 \times 10^{-3} \mathrm{M}$ phenol. Integral reflectance values were taken from Figure 11 and $\theta$ values from the capacity curve at the same concentration.

which the electric double layer is represented as two capacitors connected in parallel, with only phenol molecules contained between the plates of one of them and only supporting electrolyte between those of the other. It is worth noticing that the charge region covered by the second linear zone is characterized by a capacitance curve that shows no hysteresis on reversing the slow potential sweep, Figure 4 (inset).

In Figure 11 the third zone, with values of charge greater than $+10 \mu \mathrm{C} / \mathrm{cm}^{2}$, is characterized by the highest slope and arises primarily from the electronic properties of the metal strongly perturbed by the presence of the adsorbate. That is, the contribution of the charge to the change in reflectance is no longer independent of the electrode coverage. The process giving rise to peak II is more easily detected through differential optical measurements than by means of capacitance determinations. Therefore, it is unlikely that this transformation arises from the weakly adsorption of fluoride ion, either on top of the phenol molecule or on the metal itself (on uncovered patches), as this anion should not give rise to the strong perturbation of the spectrum of gold observed at ca. $1.7 \mathrm{eV}$. Furthermore, the charge region covered by the third linear zone is characterized by a capacitance curve that shows hysteresis on reversing the slow potential sweep, Figure 4. This behavior, typical of strongly bound adsorbates, points to the possibility that phenoxide ions be adsorbed on the electrode in this potential region.

\section{Conclusions}

The adsorption giving rise to peak $\mathrm{I}$, observed at negative charge on the metal, leads to a $\Gamma_{\max }$ of $3.0 \times 10^{-10} \mathrm{~mol} / \mathrm{cm}^{2}$ pointing to a flat orientation of phenol bonded to the surface through its $\pi$ system. The optical behavior in this potential region is similar in shape to that of bare gold, the intensity being proportional to the total charge on the metal. Besides, optical effects arising from either charge or coverage modulation can be separated out in the peak I region. The interaction between phenol and gold is stronger than that of phenol on $\mathrm{Hg}$ although not sufficiently intense to modify the intrinsic optical properties of the metal.

The adsorbate-metal interaction in the potential range of peak II is indicative of the presence of a covalent bond as suggested by the significant distortion of the electroreflectance spectra of gold which appears extended up to the red region, away from the absorption edge of bare gold.

Peak II can be associated with the reorientation of the phenol molecule from flat to vertical. In this position the 
bonding of the molecule to the surface can be thought of as taking place either through the lone pair of the oxygen atom or, at high $\mathrm{pH}$, by means of the phenoxide ion, taking into account the surface is positively charged in this potential region. Interactions between molecules in the two orientations seem to be responsible for the gradual, nonsharp, transition.

Separation of optical contributions stemming from charge and coverage is no longer possible in this zone because of the intense metal-molecule coupling. This behavior is typical of strong adsorption of anions such as halides.

Acknowledgment. Support for this project by the Consejo Nacional de Investigaciones Cientificas y Técnicas, the Comisión de Investigaciones Científicas de la Provincia de Buenos Aires, and the Regional Scientific Programme of the Organization of American States (OAS) is gratefully acknowledged.

Registry No. $\mathrm{PhOH}, 108-95-2 ; \mathrm{Au}, 7440-57-5$. 